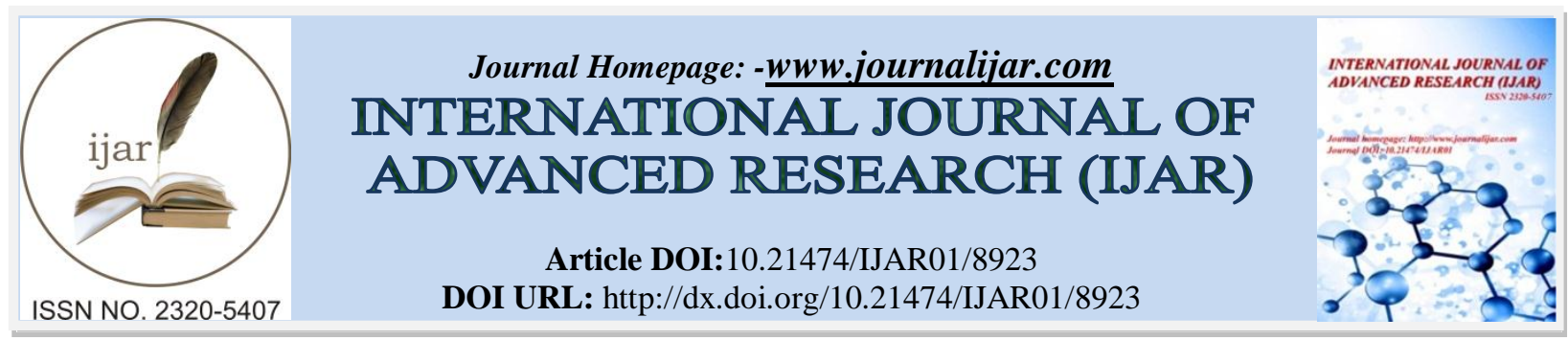

RESEARCH ARTICLE

\title{
ASSESSMENT OF URINARY KIM-1 AS RENAL INJURY MARKER IN CHRONIC KIDNEY DISEASE PATIENTS.
}

Nabila Noor, Ghazala Yasmeen, Sumera Sohail, Lubna Naz and Nazish I. Khan.

Pathophysiology Research Unit, Department of Physiology, University of Karachi.

\section{Manuscript Info}

\section{Manuscript History}

Received: 21 February 2019

Final Accepted: 23 March 2019

Published: April 2019

Key words:-

KIM1, CKD, renal injury, biomarker.

\section{Abstract}

Background: For the identification of kidney damage and chronic kidney disease stages there are certain biomarkers. Usually blood indicators are used for the diagnosis particularly creatinine \& urea requiring frequent blood sampling from the patients already facing anemia, use of these biomarkers are under high debate because of many affecting factors and cofounders. New, noninvasive more reliable markers are other consideration. Kidney Injury Molecule (KIM-1) is one of such recently reported protein identified in direct relation to renal parenchyma damage and a part of protein is excreted in urine too that is normally absent in urine samples.

Objective: The present study was planned to determine whether urinary KIM-1 level act as a better and noble biomarker of kidney functions in comparison with conventional GFR, creatinine and others in different stages of chronic kidney disease.

Methodology: The cross-sectional study was conducted for the period of one year. Urine and blood samples were collected from $100 \mathrm{CKD}$ patients of different stages. Urinary KIM-1 protein was detected by ELISA. The data were statistical analyzed using SPSS Version 17 at chosen level of significance $\mathrm{p}<0.05$.

Results: The 100 partakers mean age was 50 years comprising of $45 \%$ male gender presented with different comorbidities including diabetes mellitus (36\%), hypertension (66\%), smoking (15\%) categorized into CKD stages as stage $1(0 \%), 2(10 \%), 3(25 \%), 4(42 \%), 5(23 \%)$ respectively. Our data showed strong relationship between kidney damage and urinary KIM- 1 excretion with a correlation of -0.75 $(p<0.01)$ for stage $2,-0.6(p<0.04)$ for stage $3,-0.3(p<0.06)$ for stage 4 , $-0.7(\mathrm{p}<0.05)$ for stage 5 as calculated between baseline GFR \& KIM 1. Conclusion: KIM-1 can be used effectively to monitor the renal injury in CKD patients through urinary samples rather than taking blood samples frequently. 


\section{Introduction:-}

Chronic kidney disease (CKD) is a term that delineates every form of chronic kidney affliction and the condition in which an individual's estimated glomerular filtration rate (eGFR) is lesser than or equal to $60 \mathrm{ml} / \mathrm{min} / 1.73 \mathrm{~m}{ }^{2}$ irrespective of the causes or a urinary albumin-to-creatinine ratio $>30 \mathrm{mg} / \mathrm{g}$.

GFR and creatinine are used as a biomarker of CKD but for the early prediction of CKD progression, there is lack of sensitive and specific biomarkers as GFR value is affected by many cofounders like age, gender and authenticity. When damage start in the kidney, so not any drastic alteration can be observed in the GFR range because of the compensatory mechanism. Furthermore, the kidney's reserve nephrons are activated as a result GFR remains between normal range while the kidney damage is being continued. Serum creatinine, eGFR, and proteinuria are insensitive and reliance on these parameters may result in extensive time lapse where successful interventions could be experimented and implemented.

The recent application of advanced technologies such as functional genomics, proteomics, and bio fluid profiling has exposed numerous new candidates that are emerging as prognostic biomarkers of CKD. Among these include proteins appears in the urine for instance neutrophil gelatinase-associated lipocalin (NAGL), kidney injury molecule-1(KIM-1), and liver-type fatty acid binding protein. Moreover, a better explanation of complicated pathogenic mechanisms involved in CKD advancement has given differential markers of CKD progression that are being actively assessed. Indicators involved in this group are plasma proteins such as asymmetric dimethyl arginine, adiponectin, Apo lipoprotein A-IV, the natriuretic peptides, fibroblast growth factor (FGF), neutrophil gelatinaseassociated lipocalin, and urinary $\mathbf{N}$-acetyl- $\beta$-D-glucosaminidase (Han, 2008). Amongst the emerging biomarkers of renal damage, we evaluated the potential of KIM-1.

KIM-1 is an epithelial cell adhesion molecule also referred as T cell immunoglobulin and mucin domain 1 (TIM-1) (Waanders et.al., 2010). KIM-1 is reported to appear in the renal injury including acute kidney injury (AKI) and (CKD) originating in the $3^{\text {rd }}$ segment of proximal tubule. It is a transmembrane protein type I containing an $\mathrm{N}$ terminal immunoglobulin-like domain, mucin domain with $\mathrm{O}$ - and $\mathrm{N}$-linked glycosylations, transmembrane segment, cytoplasmic signalling domain (Kuehn, 2002). It promotes epithelial regeneration, regulates tubular cell apoptosis.

According to several studies, a sensitive and particular renal tissue insult indicator is KIM-1 as KIM-1 indicates kidney disease although lesser is explored about exact molecular phenomenon that controls such events (Bonventre, 2008). In kidney patients, KIM-1 is expressed in different disease outcomes like ischemia, drugs nephrotoxicity, CKD, acute/chronic renal transplant dysfunction Any other organ doesn't express KIM-1 to a degree that would affect renal excretion. It is also a predictor of prognosis specifically in acute kidney injury. In future KIM-1 may turn out to be a novel therapeutic marker for kidney injury of several stages.

The expression of KIM-1 gene or protein is imperceptible in normal kidney. messenger RNA of KIM-1 in the injured kidney is rapidly duplicated at different sites incliding glomerulus, peritubular interstitial cells, or inner medullary cells the expression of KIM-1 is present in the injured kidney.

KIM-1 is expressed in the dedifferentiated proximal renal tubular epithelial cells and caused damage in the tubular epithelial cells go through differentiation and proliferation, and the function of KIM-1 as a biomarker has a robust future. While the specific function of KIM-1 is still unknown but It may take part in the advancement of renal injury or repair (Venkata et al., 2014) .

Principally in CKD the protective function of KIM-1 was reported which includes ischemic or toxic damages and mainly in the chronic renal diseases the damaging function of KIM-1 was reported

So, we can assume that KIM-1 may play a protecting role in the early stage of kidney damage, and in the advance stage, it may play a destructive role due to the unnecessary cell propagation caused by the KIM- 1 induced renal repair. 
Urine KIM-1 level had been confirmed to be closely related to tissue KIM-1 level and to compare with kidney tissue damage (Yin \& Wang, 2016).

There are various characteristics of KIM-1 making it a perfect biomarker for kidney injury. For instant, KIM-1 is not expressed in normal kidney but specifically expressed in injured proximal tubular cells, and such an expression can continue till the damaged cells have entirely well again. Furthermore, the rapid and integrated cleavage of its ectodomain into the lumens of kidney tubules can make it measurable in urine. Urinary KIM-1 level is strictly related to tissue KIM-1 and associates with the severity of renal damage, so quantitation of urinary KIM-1 is likely to be a non-invasive and sensitive method for the evaluation of kidney injury and even for monitoring the beneficial effects of kidney injury. In the study of nephrotoxicity, urinary KIM-1 levels amplified severely prior than the increases of blood urea nitrogen and plasma creatinine (ziyue et al, 2016). In the renal transplant surgeries, KIM-1 appearance was reported being able to detect early tubular damage that was not obvious by histologic investigation, and KIM-1 also helped in discrimination of acute tubular necrosis from another allograft dysfunction. Therefore, KIM-1, act as a specific and primary indicator of tubular injury, may be used to guide and individualize renoprotecting intervention (Branislava et al, 2015). A highly sensitive and specific urinary biomarker for chronic kidney injury is KIM-1 and for various kidney disease it may also be a therapeutic target. The objective of present study is to assess the urinary KIM-1 as a kidney damage marker for chronic kidney disease.

\section{Methodology:- \\ Study Design:}

A paper-based questionnaire was used for systemic data collection including questions regarding patient's history, family history, height, weight, risk factors comorbidities \& co-factor. Data was collected from 100 CKD patients meeting the criteria. All CKD patients were considered excluding pregnant females, addicts, individual who have plus 70 age and end stage renal diseases, liver disease, hepatitis, AIDS and any other disorders which are contiguous.

Blood and urine samples were collected from all partakers. Urine samples were centrifuged 2000rpm for 5 min to remove cellular components and debris and the supernatant was stored at $-80^{\circ} \mathrm{C}$ until used for biochemical assay. All urine samples were initially tested through urinary dipstick prior to centrifugation. From blood sample creatinine was measured by the Bartel method for GFR estimation.

Urine samples were used for estimation of KIM-1 using ELISA kit following manufacturer's instruction. Briefly a monoclonal antibody specific for human KIM-1 had been pre-coated onto a microplate. Standards and samples were pipetted into the wells and any KIM1 present was bound by the immobilized antibody. After removing free unbound particles, an enzyme-linked polyclonal antibody specific for human KIM-1 was poured to the wells, followed by again washing a substrate solution was poured to the wells resulting into colour development in proportion to KIM-1 level bound in the first step, stopper was added, and colour intensity was measured.

Statistical analyses were performed using SPSS Version 17. applying t-test and spearman's correlation. P-values less than 0.05 were considered statistically significant.

The purpose of study was clearly explained to all participants; assured personal privacy, interview was conducted in national language along with a written consent. Throughout the study the principles mentioned in Helenski protocol were followed.

\section{Result:-}

The study comprised of 100 samples including $45 \%$ male and 55\% female with mean age of 50 years and standard deviation 14. The most prevailed comorbidity found in CKD patients was hypertension (66\%) followed by diabetes mellitus $(36 \%)$ while $15 \%$ were found current smokers $17 \%$ former smokers, $35 \%$ passive smokers and $33 \%$ patients consuming oral tobacco.

The common causes of chronic kidney disease were diabetes miletus, Hypertension; Chronic Glomerulonephritis (CGN) and fewer were systemic erythematous, nephritis and nephrotic. 
None of the participant lies in CKD stage 1, $10 \%$ in stage 2, 25\% in stage 3, $42 \%$ in stage 4 while $23 \%$ resided in stage 5 .

\begin{tabular}{|l|l|l|l|l|}
\hline $\begin{array}{l}\text { CKD } \\
\text { Stage }\end{array}$ & Baseline eGFR & $\begin{array}{l}\text { KIM-1 } \\
\text { ng/ml }\end{array}$ & R-values & p-value \\
\hline 2 & $\mathbf{8 5} \pm 3.5$ & $\mathbf{3 . 4} \pm 0.8$ & -0.75 & 0.01 \\
\hline 3 & $64.6 \pm 4.8$ & $6.2 \pm 2.1$ & -0.6 & 0.04 \\
\hline 4 & $38 \pm 8.4$ & $6.8 \pm 2.9$ & -0.3 & 0.06 \\
\hline 5 & $22.9 \pm 3.9$ & $\mathbf{8 . 2} \pm 3.4$ & -0.7 & 0.05 \\
\hline
\end{tabular}

Table 1:-The relationship between GFR and urinary Kidney Injury Molecule-1 in different stages of chronic kidney disease

The result showed that there is a strong relationship between kidney functional capacity deterioration and expression of KIM-1 in urine that also found statistically significant as compared between baseline eGFR and the urinary KIM1 concentration. In stage 2 CKD patients, the correlation between GFR and KIM- 1 was found -0.75 attaining level of statistical significance $(\mathrm{p}<0.01)$; advancing to stage 3 this relationship remained negatively strong and significant too $(\mathrm{p}<0.04)$ but stepping to stage 4 with further decline in GFR the increase in urinary KIM-1 was not linear $(r=-$ $0.3)$ and found statistically non-significant $(\mathrm{p}<0.06)$ that again became significant when assessed in stage 5 patients $(\mathrm{p}<0.05)$ as shown in Table 1.

\section{Discussion:-}

CKD is a chronic disease that remained asymptomatic before advanced to second stages, so mostly individuals suffering with CKD are diagnosed in later stages with disease progressed enough that few opportunities are left to prevent complications (Kinchen, 2002). As a matter of fact, earlier diagnosis would provide sufficient time for investigation and suitable remedy but would have to develop more focused reliable evaluation options for high risk yet asymptomatic patients.

Currently, CKD is conventionally diagnosed through serum creatinine levels and estimation of GFR. but, creatinine is an less reliable marker specially in the phase of acute changes. The major drawback of creatinine is that we need all the time blood sample of the patient and we know that all the patient with CKD have low level of blood in their body and they are anaemic, and prognosis for kidney disease progression or CVD; and GFR estimates. The glomerular filtration rate (GFR) is conventionally taken as ideal reflector of renal functional capacity in humans. But GFR is challenging to estimate exactly in clinical routines, usually clinicians calculate GFR using serum levels of creatinine. However, the calculated value is less accurate because there are other factors too affecting creatinine other than creatinine filtration. It is recommended that GFR measures alone should not be practiced for follow-up of CKD; approximately fifty percent imagined practical issues with daily record keeping; a general consensus that frequent regular measure of GFR lead to frequent appointment; commonly used Cockcroft-Gault formula, though information of the elements with suboptimal validity of equation; and the protocols followed for creatinine assay in local laboratories were uncertain.( Levey, et. al, 2005).

Furthermore, serum creatinine levels can vary widely with age, gender, lean muscle mass, muscle metabolism, and hydration status. Secondly, serum creatinine concentrations may not change until about $50 \%$ of kidney function has already been lost. Third, at lower rates of glomerular filtration, the amount of tubular secretion of creatinine results in overestimation of renal function. Finally, during acute changes in glomerular filtration, serum creatinine does not accurately depict kidney function until steady-state equilibrium has been reached, which may require several days. (Han et al, 2005). So, we use KIM-1 concentration in urine for the identification of CKD.

The first human subject studies with KIM-1 were published in 2002 (Han, et al. 2002) reporting profoundly enhanced expression of KIM-1 renal biopsy samples collected from acute tubular necrosis patients for diagnostic purpose, and markedly increased KIM-1 ectodomain concentration in urine samples of acute kidney injury patients even before appearance of casts in urine, KIM 1 levels were in proportion to intensity of AKI tracking severity of disease as measured by peak serum creatinine value, subsequently showed that KIM-1 is a sensitive biomarker for renal insult (Han, et. al, 2008). 
In non-diabetic CKD patients, urinary KIM-1 found elevated in protein uric and was decreased if the proteinuric patients were treated with renin-angiotensin-aldosterone inhibition, sodium restriction and or diuretic therapies that reduced the proteinuria, thus linking the degree of proteinuria to proximal tubule injury as quantitated by KIM1 in the urine (Waanders, et. al., 2010)

In 2009, wander, et. al did a research and they reported that the proteinuria to proximal tubular injury as quantised by KIM-1 in the urine. We have the same finding in favour of the result and supported by the study another study which is reported by Vaidya, et.al. (2006) stated that the development of a quantitative assay for measuring KIM-1 in the rat urine as a biomarker for AKI and demonstrate that marked increases in urinary KIM-1 can be measured under conditions where changes in other standard indicators are not measurable. These results have direct implications for evaluation of nephrotoxicity in animals where sensitive and specific measures of toxicity have been elusive. Nephrotoxicity often is evaluated by reliance on serum creatinine levels, an insensitive measure of kidney injury, or histological analysis, which requires killing many animals and is inherently difficult to quantitate.

KIM-1 can be recommended as potential marker for investigation of renal insult on various basis, summarized into three core causes as KIM 1

1. not demonstrable in healthy kidney.

2. expressed by proximal tubular segments affected by ischemic or toxic insult

3. ectodomain is shed down from insulted cells, appeared into urine within 12 hour (Bonventre, 2009)

This study demonstrates that urinary KIM-1 protein concentration is significantly higher in urine samples from patients with CKD and it is a potent biomarker of the kidney function. So, the result led to more reliable indicator of pathogenesis and highlight the significance of urinary KIM-1.

Further follow up potential projects are in development to explain various renal disease mechanisms for identification of reliable urinary renal biomarkers that will replace invasive renal tissue biopsy. The study limitations are smaller sample size and lack of follow up, further studies are suggested to investigate KIM-1 sensitivity and specificity as clinical biomarker.

\section{Conclusion:-}

In conclusion, urinary KIM-1 can be helpful in the assessment of renal damage in CKD patients as more reliable biomarker than the practiced plasma creatinine and GFR.

\section{Conflict of Interest:}

Authors declare no conflict of interest.

\section{References:-}

1. Bonventre JV. Kidney Injury Molecule-1 (KIM-1): a specific and sensitive biomarker of kidney injury. Scand J Clin Lab Invest Suppl. 2008;241:78-83. doi: 10.1080/00365510802145059.

2. Branislava Medić, Branislav Rovčanin, Gordana Basta Jovanović, Sanja Radojević-Škodrić, and Milica Prostran. Kidney Injury Molecule-1 and Cardiovascular Diseases: From Basic Science to Clinical Practice. B. BioMed Research International Volume 2015, http://dx.doi.org/10.1155/2015/854070.

3. Han W, Waikar S, Johnson A, Betensky R, Dent C, Devarajan P, et al. Urinary biomarkers in the early diagnosis of acute kidney injury. Kidney international. 2008;73(7):863-9.

4. Han WK, Alinani A, Wu CL, Michaelson D, Loda M, McGovern FJ, et al. Human kidney injury molecule-1 is a tissue and urinary tumor marker of renal cell carcinoma. Journal of the American Society of Nephrology : JASN. 2005;16(4):1126-34.

5. Han WK, Bailly V, Abichandani R, Thadhani R, Bonventre JV. Kidney Injury Molecule-1 (KIM-1): a novel biomarker for human renal proximal tubule injury. Kidney international. 2002;62(1):237-44.

6. Kinchen KS, Sadler J, Fink N, Brookmeyer R, Klag MJ, Levey AS, et al. The timing of specialist evaluation in chronic kidney disease and mortality. Annals of internal medicine. 2002;137(6):479-86.

7. Kuehn EW, Park KM, Somlo S, Bonventre JV. Kidney injury molecule-1 expression in murine polycystic kidney disease. Am J Physiol Renal Physiol. 2002 Dec;283(6):F1326-36. 
8. Levey AS, Eckardt KU, Tsukamoto Y, Levin A, Coresh J, Rossert J, et al. Definition and classification of chronic kidney disease: a position statement from Kidney Disease: Improving Global Outcomes (KDIGO). Kidney international. 2005;67(6):2089-100.

9. Vaidya VS, Ramirez V, Ichimura T, Bobadilla NA, Bonventre JV. Urinary kidney injury molecule-1: a sensitive quantitative biomarker for early detection of kidney tubular injury. American Journal of PhysiologyRenal Physiology. 2006;290(2):F517-F29.

10. Venkata S. Sabbisetti, Sushrut S. Waikar, Daniel J. Antoine, Adam Smiles, Chang Wang, Abinaya Ravisankar, Kazumi Ito, Sahil Sharma, Swetha Ramadesikan, Michelle Lee, Rebeccah Briskin, Philip L. De Jager, Thanh Thu Ngo, Mark Radlinski, James W. Dear, Kevin B. Park, Rebecca Betensky, Andrzej S. Krolewski and Joseph V. Bonventre. Blood Kidney Injury Molecule-1 Is a Biomarker of Acute and Chronic Kidney Injury and Predicts Progression to ESRD in Type I Diabetes. JASN October 2014, 25 (10) 2177-2186; DOI: https://doi.org/10.1681/ASN.2013070758.

11. Waanders F, van Timmeren MM, Stegeman CA, Bakker SJ, van Goor H. Kidney injury molecule-1 in renal disease. J Pathol. 2010 Jan;220(1):7-16. doi: 10.1002/path.2642.

12. Yin C, Wang N. Kidney injury molecule-1 in kidney disease. Ren Fail. 2016 Nov;38(10):1567-1573. doi: 10.1080/0886022X.2016.1193816.

13. Ziyue Li, Chuan Shen, Yadong Wang, Wei Wang, Qian Zhao, Zhenzhong Liu, Yang Wang, and Caiyan Zhao. Circulating kidney injury molecule-1 is a novel diagnostic biomarker for renal dysfunction during long-term adefovir therapy in chronic hepatitis. Medicine (Baltimore). 2016 Nov; 95(44): e5264. 